\title{
CD200 expression in mantle cell lymphoma identifies a unique subgroup of patients with frequent IGHV mutations, absence of SOX11 expression, and an indolent clinical course
}

\author{
Zhihong $\mathrm{Hu}^{1}$, Yi Sun ${ }^{1}$, Ellen J Schlette ${ }^{1}$, Guilin Tang ${ }^{1}$, Shaoying $\mathrm{Li}^{1}$, Jie $\mathrm{Xu}^{1}$, \\ C Cameron Yin ${ }^{1}$, Ken H Young ${ }^{1}$, Keyur Pravinchandra Patel ${ }^{1}$, Roberto N Miranda ${ }^{1}$, \\ Maitrayee Goswami ${ }^{1}$, Michael Wang ${ }^{2}$, Jeffrey L Jorgensen ${ }^{1}$, L Jeffrey Medeiros ${ }^{1}$ and \\ Sa A Wang ${ }^{1}$ \\ ${ }^{1}$ Department of Hematopathology, The University of Texas MD Anderson Cancer Center, Houston, TX, USA \\ and ${ }^{2}$ Department of Lymphoma and Myeloma, The University of Texas MD Anderson Cancer Center, \\ Houston, TX, USA
}

\begin{abstract}
CD200, a marker currently utilized in the diagnosis of B-cell lymphoma, is uniformly positive in chronic lymphocytic leukemia/chronic lymphocytic leukemia, and is usually absent in mantle cell lymphoma. Over a 6 year-period, of 668 mantle cell lymphoma assessed by flow cytometry, CD200 expression was detected in 25 patients $(\sim 4 \%)$. All 25 patients had bone marrow involvement; however, $11(44 \%)$ patients had no nodal or extranodal disease and belonged to non-nodal leukemic variant mantle cell lymphoma. Morphologically, bone marrow showed an unusual interstitial infiltrative pattern in 14/25 (56\%) and small round cells resembling chronic lymphocytic leukemia in 9/25 (36\%). CD23 was positive in 19/25 (76\%) patients; and SOX11 was only positive in $5 / 21(24 \%)$. All 4 patients tested showed IGHV mutations. With a median follow-up of 23 months, $12 / 24(50 \%)$ patients were not treated. These clinicopathological features were significantly different from 154 randomly chosen CD200-negative mantle cell lymphoma patients, in SoX11 positivity $(24 \%$ versus $74 \%, P<0.0001)$, CD23 expression $(76 \%$ versus $8 \%, P<0.0001)$, a non-nodal leukemic presentation $(44 \%$ versus $2 \%, P<0.001)$, and therapy requirement $(50 \%$ versus $92 \%, P<0.0001)$. This is the first study to show that CD200 expression in mantle cell lymphoma, though uncommon, identifies a subgroup of mantle cell lymphoma patients with characteristic pathological features, frequent non-nodal leukemic variant, and an indolent clinical course. Modern Pathology (2018) 31, 327-336; doi:10.1038/modpathol.2017.135; published online 6 October 2017
\end{abstract}

The World Health Organization (WHO) classification defines mantle cell lymphoma as a B-cell lymphoma with t(11;14)(q13;q32), in which CCND1 at chromosome 11q13 is juxtaposed with the immunoglobulin heavy chain $(I G H)$ at $14 \mathrm{q} 32$ on the derivative chromosome 14. The CCND1-IGH fusion results in overexpression of cyclin D1 protein. ${ }^{1}$ Mantle cell lymphoma is most often an aggressive B-cell neoplasm with a median overall survival of 3 years and is considered incurable using conventional chemotherapeutic approaches. However, it has been increasingly recognized that mantle cell lymphoma

Correspondence: Dr SA Wang, MD, Department of Hematopathology, The University of Texas MD Anderson Cancer Center, 1515 Holcombe Blvd, Unit 72, Houston, TX 77030, USA.

E-mail: swang5@mdanderson.org

Received 26 June 2017; revised 31 August 2017; accepted 31 August 2017; published online 6 October 2017 as currently defined is more heterogeneous in its morphological and immunophenotypic features, molecular genetic characteristics, and outcomes. ${ }^{2-6}$ Some mantle cell lymphoma patients may have a long, clinically silent latency period or a remarkably indolent disease course. ${ }^{6}$ In accord with these findings, recently two indolent variants of mantle cell lymphoma, in situ mantle cell neoplasia and non-nodal leukemic variant mantle cell lymphoma, have been recognized in the 2016 revision of the WHO classification of lymphoid neoplasms. ${ }^{7}$

CD200 (OX-2 antigen), a type I immunoglobulin superfamily membrane protein, is composed of a light chain-like structure with two extracellular variable- and constant-like domains, a transmembrane segment and a cytoplasmic tail. ${ }^{8}$ CD200 is expressed in a subset of $\mathrm{T}$ cells and CD19+ B lymphocytes, dendritic cells and is highly expressed in central and peripheral nerve tissue. ${ }^{9}$ CD200 is 
expressed at various levels in B-cell and plasma cell neoplasms. ${ }^{6,9-15}$ Among the CD5+ B-cell neoplasms, CD200 is uniformly positive in chronic lymphocytic leukemia/chronic lymphocytic leukemia including atypical chronic lymphocytic leukemia, ${ }^{16,17}$ whereas, its expression in mantle cell lymphoma is very unusual.16,17 As a result, CD200 has been used as an important marker in the differential diagnosis between mantle cell lymphoma and atypical chronic lymphocytic leukemia.

In our laboratory, we have incorporated the use of CD200 in our clinical flow cytometry testing in 2011, and applied to biopsies, bone marrow and peripheral blood samples submitted for the workup of B-cell lymphomas. We have observed CD200 expression occasionally in cases of mantle cell lymphoma that immunophenotypically resemble chronic lymphocytic leukemia. However, the biological significance of CD200 in mantle cell lymphoma is not known. We were aware of a recent study by Espinet and colleagues $^{18}$ describing an entity 'monoclonal asymptomatic lymphocytosis, cyclin D1-positive' which showed with frequent expression of CD200. But this type of disease was thought to be a different entity from mantle cell lymphoma. In this study, we retrospectively reviewed CD200 expression in a large series of mantle cell lymphoma patients to determine the frequency as well as the clinicopathological features associated with CD200+ mantle cell lymphoma cases.

\section{Materials and methods}

\section{Case Selection}

Starting in 2011, CD200 has been integrated into the flow cytometry antibody panel for the study of B-cell lymphoproliferative disorders in our clinical flow cytometry unit. For this study, cases of mantle cell lymphoma analyzed by flow cytometry immunophenotyping over an interval of 6 years (May 2011 through December 2016) were retrospectively reviewed. All cases of mantle cell lymphoma were confirmed by cyclin D1 overexpression assessed by immunohistochemistry and/or CCND1/IGH by fluorescence in situ hybridization (FISH) or $\mathrm{t}(11 ; 14)(\mathrm{q} 13$; q32) by conventional karyotyping analysis. The histological sections of all CD200+ mantle cell lymphoma cases were also retrospectively reviewed by at least two more hematopathologists. Demographic information, clinical data including clinical disease manifestations, treatment regimens and clinical follow-up information were collected and included. This study was approved by the Institutional Review Board of our hospital.

\section{Flow Cytometry Immunophenotyping}

For flow cytometry immunophenotypic studies, samples (lymph node, extranodal site, bone marrow or peripheral blood) were incubated with monoclonal antibodies for $10 \mathrm{~min}$ at $4^{\circ} \mathrm{C}$, followed by erythrocyte lysis with ammonium chloride (PharmLyse, BD Biosciences, San Diego, CA, USA) at room temperature for $10 \mathrm{~min}$ using a standard lyse/wash technique. Samples were acquired on FACSCanto II instruments (BD Biosciences). Antibody panels are shown in Supplementary Table 1. A total of 100000 events were acquired for each tube. Data were analyzed using FCS Express software (De Novo Software, Los Angeles, CA, USA). Lymphocytes were defined by CD45/SSC (side scatter) characteristics. CD200 expression was assessed on CD19+CD5 + aberrant B-cell population, with T cells as internal negative controls as well as fluorescence minus one control. CD200 was considered positive when at least $20 \%$ of lymphoma cells had the expression, an arbitrary cutoff used to define a meaningful expression in most of the biomarker studies.

\section{Histological Examination and Immunohistochemistry Results}

Hematoxylin-eosin stained slides of lymph node, extranodal sites, and bone marrow core biopsy specimens were reviewed. Wright Giemsa stain was performed on bone marrow aspirate and peripheral blood smears. A bone marrow differential count was performed by counting 500 nucleated cells on aspirate smears.

Immunohistochemical studies were performed using formalin-fixed paraffin-embedded tissue sections as described previously. ${ }^{19}$ Antibodies and dilutions used in this study included: CD20 (dilution 1:400), cyclin D1 (1:350), and p53 (1:200) (Dako, Carpinteria, CA, USA); CD5 $(1 ; 20)$ (ThermoScientific, Fremont, CA, USA); Myc (1:200) (Ventana, Tucson, AZ, USA); SOX11 (1:200) (Cell Marque, St. Louis, MO, USA); PAX5 (1:35) (BD Biosciences); and LEF1 (1:100) (Abcam, Cambridge, MA, USA).

\section{Conventional Karyotyping, FISH Analysis, and IGH Somatic Hypermutation Analysis}

Conventional G-banded karyotypic analysis was performed using standard methods as described previously. ${ }^{20}$ Karyotypes were analyzed from 24-h unstimulated and/or 72-h lipopolysaccharides stimulated cultures of bone marrow aspirate specimens. At least 20 metaphases were analyzed whenever applicable. The karyotypic results were reported according to the International System for Human Cytogenetic Nomenclature 2013. ${ }^{21}$

Fluorescence in situ hybridization (FISH) was performed on bone marrow smears or on $4-\mu \mathrm{M}$ thick tissue sections of formalin-fixed paraffin-embedded, non-decalcified specimens according to the laboratory standard protocols. LSI IGH/CCND1 dual color dual fusion translocation probe (Abbott Molecular, Downers, IL, USA) was used in this study. At least 

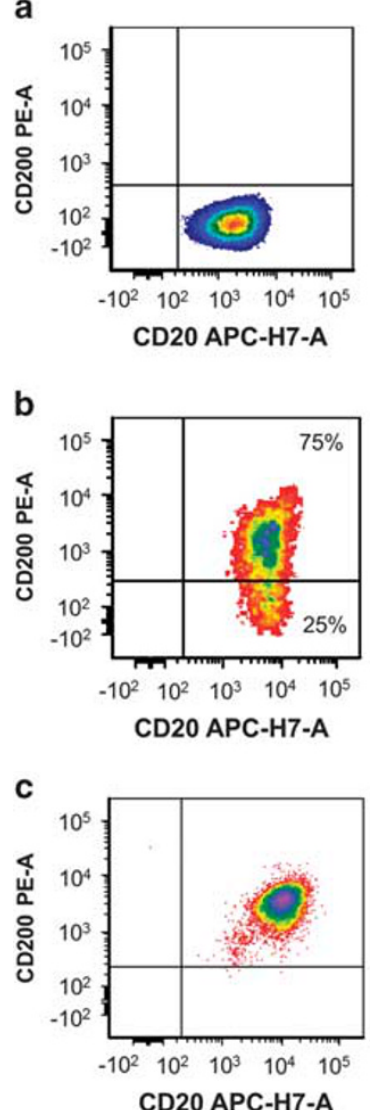
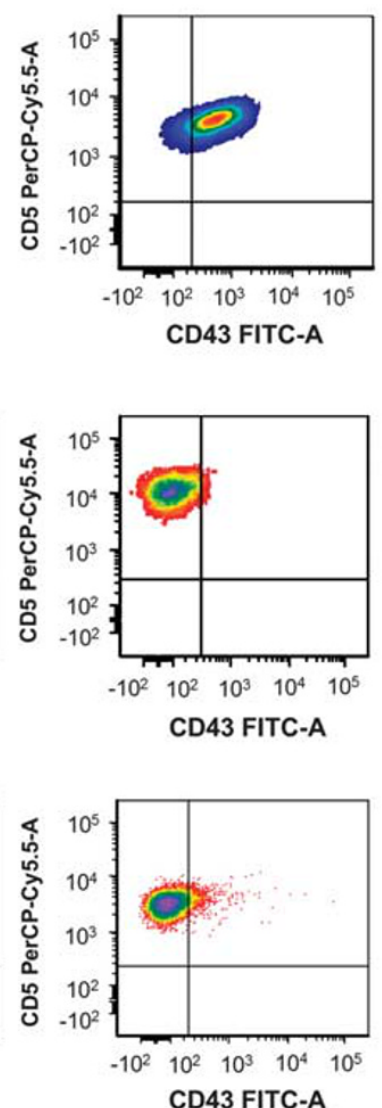
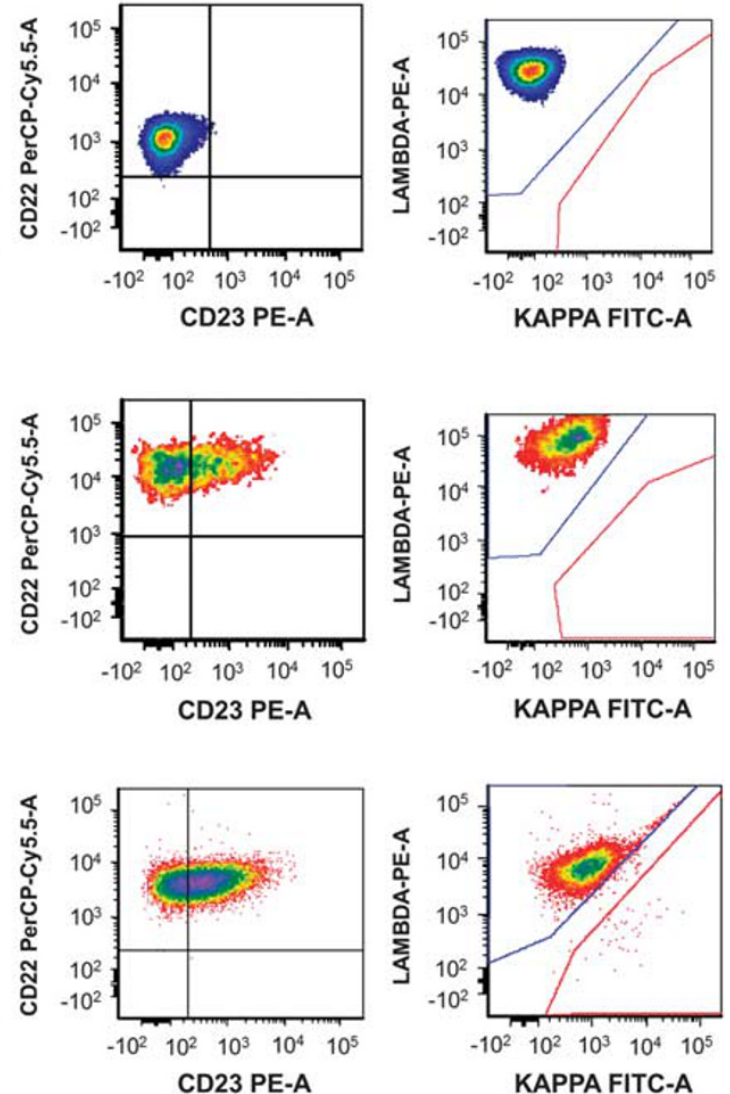

Figure 1 Examples of CD200 expression in mantle cell lymphoma by flow cytometry immunophenotyping analysis. (a) A classic mantle cell lymphoma case negative for CD200 (97\% cases). Lymphoma cells are CD5+, CD43+, CD23 -, lambda+. (b,c) Two cases of mantle cell lymphoma are positive for CD200 expression, with a partial expression pattern (b) and a uniform expression pattern (c), respectively. These two CD200+ mantle cell lymphoma cases also show partial CD23+, CD43 - , and lambda+.

200 interphase nuclei were analyzed. Based on our laboratory established threshold, the cutoff for a positive CCND1/IGH was $2 \%$ fusion signals.

IGH somatic hypermutation analysis was performed on fresh peripheral blood or bone marrow samples. RNA was extracted and multiplex PCR amplification of $I G H$ transcripts was performed using consensus variable segment primers. Sanger sequencing of the PCR products was performed and compared to germ line IGH sequences to calculate the percentage of mutated bases. The presence of $\geq 2 \%$ variation in the sequenced $\mathrm{V}$-segment of clonal IGH sequences compared to control germ line sequence was considered to be positive for somatic hypermutation. The test sensitivity was $20 \%$.

\section{Data Analysis}

For this study the mantle cell lymphoma international prognostic index was calculated using the link https://qxmd.com/calculate/mipi-mantle-cell-lym phoma-prognosis. The prognostic scores were used to determine the risk group as has been described previously. ${ }^{22,23}$

Data for categorical variables were described as the number of patients if not otherwise specified. Data for continuous variables were described as median and range. Fisher's exact test and univariate survival analysis were performed when applicable, using the statistical software of GraphPad Prism 6 (GraphPad Software, San Diego, CA, USA). Multivariate survival analysis was performed using IBM SPSS Statistics 24 (Armonk, NY, USA). Significance level was determined when the $P$-value is $<0.05$ for all analyses.

\section{Results}

\section{Patients and CD200 Expression by Flow Cytometry Immunophenotyping}

From a total of 668 mantle cell lymphoma patients assessed by flow cytometry immunophenotyping, CD200 was positive in 25 ( $4 \%)$ patients (Figure 1a). bone marrow was involved in all 25 patients and the median of lymphoma cells detected by flow cytometry immunophenotypinG was 16\% (range, $<1-$ $66 \%$ ) of total nucleated cells. CD200 expression was uniformly positive in $8(32 \%)$ patients and partial positive in 17 (68\%) patients. Examples of CD200 partial and uniform positive expression are shown in Figure $1 \mathrm{~b}$ and 1c. The partial expression group 
showed a median CD200 expression of $40 \%$ positive cells (range, 20-75\%). Ten of these patients also had peripheral blood samples studied by flow cytometry immunophenotyping; of which 9 were positive for mantle cell lymphoma involvement and all 9 samples were positive for CD200 expression showing a median expression level of $42 \%$ positive cells (range, 15-100\%). Of all 668 patients, we reviewed 60-70 patients who had both bone marrow and lymph node/extranodal tissues involved by lymphoma. Two cases were found positive for CD200; and these two patients also had CD200 expression on their bone marrow lymphoma cells. CD200 was negative in both types of specimens in the remaining cases. The clinical, laboratory and pathology information of these 25 patients are shown in Table 1.

Other flow cytometry immunophenotyping results in the group of CD200+ mantle cell lymphoma cases were as follows. With a positive expression defined as $20 \%$ or more cells expressing a specific marker, CD5 was positive in 24/25 (96\%) cases, CD10 in 1/25 $(4 \%)$ and CD43 in 5/25 (20\%). CD23 was positive in $19 / 25$ (76\%), uniformly expressed in 8 and partially in 17 cases. Surface immunoglobulin light chain expression was assessed in 24 cases, and 8 (33\%) cases were monotypic kappa and 16 (67\%) cases lambda. Nine of these patients had more than one follow-up biopsies, CD200 expression was consistently detected.

\section{Clinical Features}

The clinical characteristics of these 25 patients are shown in Table 1 . In brief, there were 19 men and 6 women with a median age of 63 years (range, 36-79) at time of initial diagnosis of mantle cell lymphoma. Only 3 patients presented with constitutional symptoms, including fatigue, night sweats or fever. A complete blood count showed that $9(36 \%)$ patients had leukocytosis, 6 (25\%) lymphocytosis, 5 (21\%) anemia, and 11 (46\%) thrombocytopenia.

Eleven patients $(44 \%)$ had peripheral blood and bone marrow involvement but no lymphadenopathy or disease in gastrointestinal or other extranodal site; seven of these 11 (63\%) patients also had splenomegaly, which was also shown by PET-CT. The remaining 14 patients presented with stage IV disease, with frequent involvement of lymph nodes $(n=11)$, gastrointestinal tract $(n=5)$, peripheral blood $(n=3)$, skin $(n=1)$ and tonsil $(n=1)$.

\section{Morphology and Immunohistochemistry}

We assessed cytomorphologic features of the mantle cell lymphoma cells in bone marrow aspirate smears of 23 cases in which lymphoma cells were present in sufficient numbers for assessment. In two patients (case \#14 and \#16), the neoplastic cells represented $5 \%$ of total bone marrow cellular elements and cytomorphology assessment was difficult. In 9 (39\%)
Table 1 Demographic and clinicopathologic features of patients of mantle cell lymphoma with CD200 expression

\begin{tabular}{|c|c|}
\hline \multicolumn{2}{|l|}{ Gender (\%, n) } \\
\hline Female & $24 \%(6 / 25)$ \\
\hline Male & $76 \%(19 / 25)$ \\
\hline Age at diagnosis (years) & $63(36-79)$ \\
\hline Constitutional symptoms & $12 \%(3 / 25)$ \\
\hline \multicolumn{2}{|l|}{ Involved sites $(\%, \mathrm{n})$} \\
\hline Bone marrow & $100 \%(25 / 25)$ \\
\hline Peripheral blood & $56 \%(14 / 25)$ \\
\hline Lymph node & $44 \%(11 / 25)$ \\
\hline Spleen & $40 \%(7 / 25)$ \\
\hline GI tract & $21 \%(5 / 24)$ \\
\hline Skin & $4 \%(1 / 25)$ \\
\hline Tonsil & $4 \%(1 / 25)$ \\
\hline \multicolumn{2}{|l|}{ Complete blood count* $(\%, \mathrm{n})$} \\
\hline Leukocytosis & $36 \%(9 / 25)$ \\
\hline Lymphocytosis & $24 \%(6 / 25)$ \\
\hline Anemia & $21 \%(5 / 24)$ \\
\hline Thrombocytopenia & $46 \%(11 / 24)$ \\
\hline \multicolumn{2}{|l|}{ Bone marrow } \\
\hline \multicolumn{2}{|l|}{ Morphology } \\
\hline Classical & $40 \%(10 / 25)$ \\
\hline Small mature round & $52 \%(13 / 25)$ \\
\hline Blastoid & $8 \%(2 / 25)$ \\
\hline Neoplastic infiltrate & $16 \%(1 \%-66 \%$ \\
\hline \multicolumn{2}{|l|}{ Involvement pattern } \\
\hline Interstitial & $56 \%(14 / 25)$ \\
\hline Nodular & $20 \%(5 / 25)$ \\
\hline Nodular and interstitial & $24 \%(6 / 25)$ \\
\hline
\end{tabular}

GI, gastrointestinal. *Leukocytosis: $>10.5 \times 10^{9} /$; lymphocytosis: $>4.0 \times 10^{9} /$ l; anemia: < $12 \mathrm{~g} / \mathrm{dl}$; thrombocytopenia: < $150 \times 10^{9} / \mathrm{l}$.

cases, the mantle cell lymphoma cells were small with round nuclear contours, mature chromatin and a small amount of agranular cytoplasm with resemblance to chronic lymphocytic leukemia cells (Figure 2a). In $11(48 \%)$ cases, some mantle cell lymphoma cells showed classical morphologic features (Figure 2b) with indented/buttock nucleus and fine chromatin. In $2(9 \%)$ cases, the mantle cell lymphoma cells exhibited blastoid morphology (Figure 2c) and showed reticular chromatin and small 1 to 3 nucleoli.

Bone marrow biopsy and/or clot section showed a median lymphoma involvement of $15 \%$ (range, 5$70 \%)$. The mantle cell lymphoma was present exclusively in an interstitial growth pattern in 14 $(56 \%)$ cases (Figure 3a), a mixed interstitial and nodular pattern in $5(20 \%)$ cases; and a nodular pattern in $6(24 \%)$ (Figure 3b).

Immunohistochemistry was performed on bone marrow biopsy or clot specimen. Cyclin D1 was positive in all 25 cases. SOX11 was positive in 5/21 $(24 \%)$ cases assessed, including strongly expression in 3 cases (including 2 blastoid mantle cell lymphoma), and only seen in a small $(<10 \%)$ subset of lymphoma cells in 2 cases. P53 stain was performed in one case of blastoid variant (patient \#25) and was 

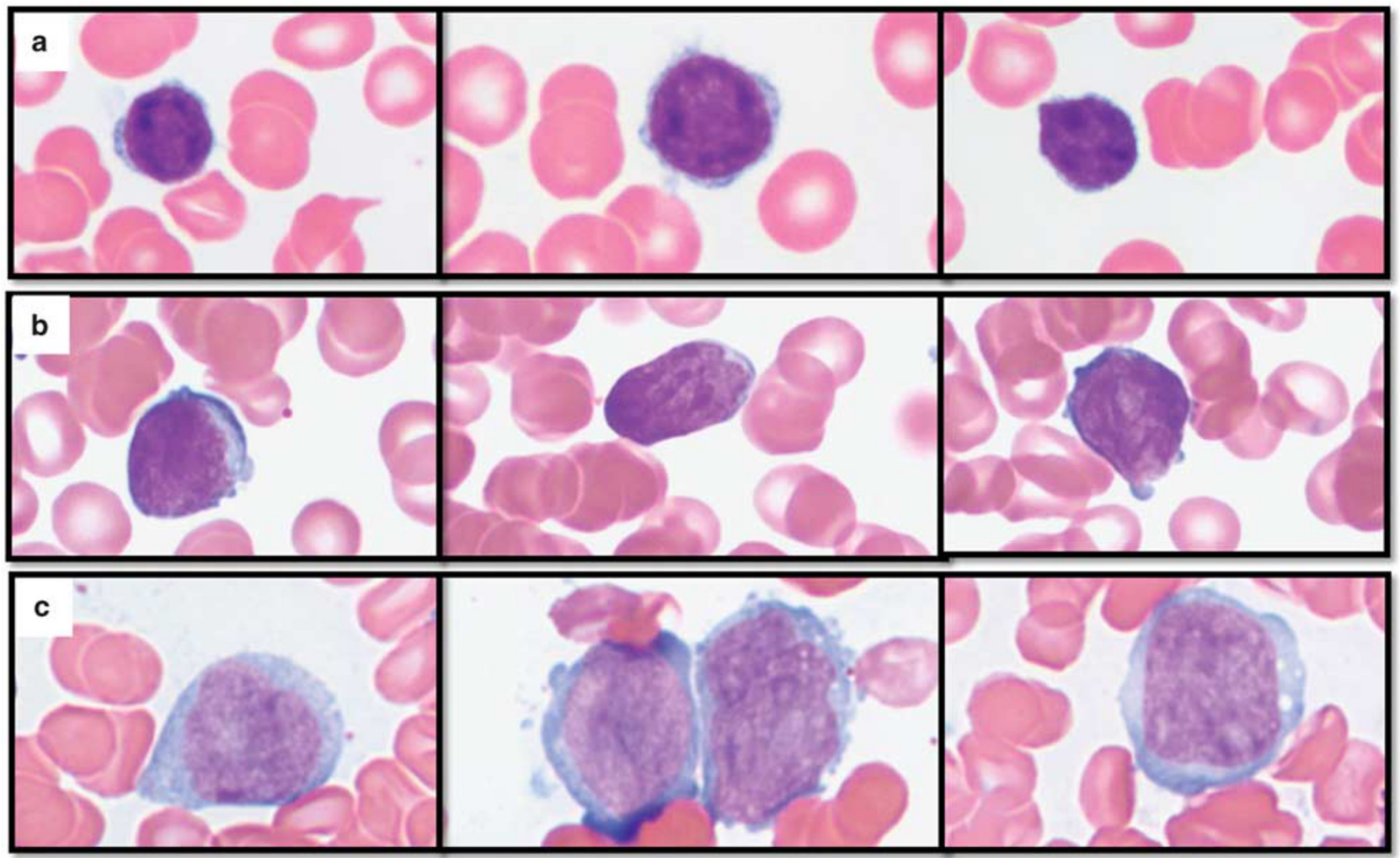

Figure 2 Morphological spectrum of mantle cell lymphoma with a positive CD200 expression on bone marrow aspirate smears. (a) Small mature cells with round nuclear contours. (b) Classic mantle cell lymphoma cells with nuclear irregularity and nuclear clefts. (c) Blastoid morphology with open chromatin and multiple small indistinct nucleoli. Wright Giemsa stain, oil immersion, magnification $\times 1000$.

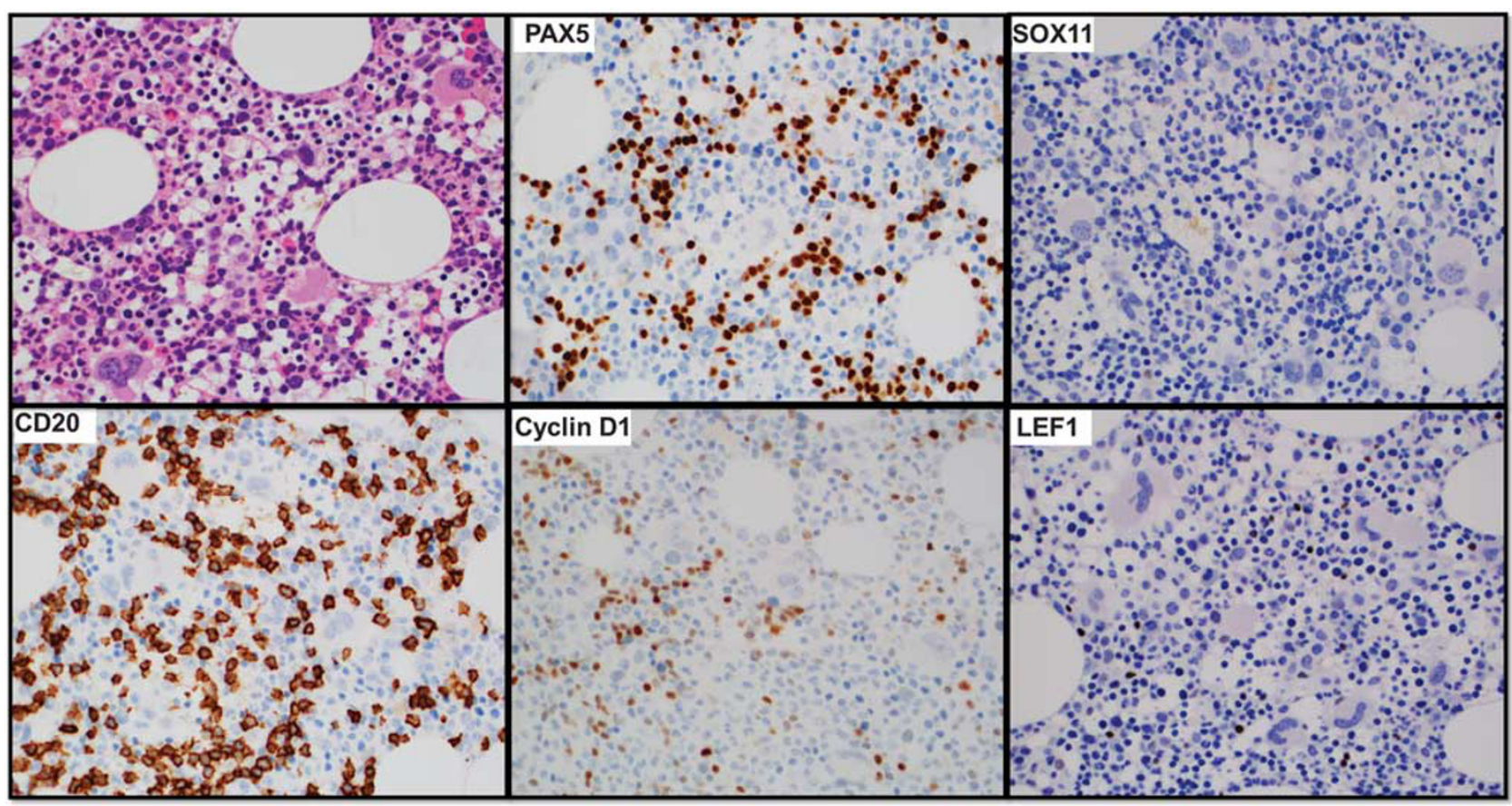

Figure 3 Bone marrow involvement by CD200+ mantle cell lymphoma. A case of CD200-positive mantle cell lymphoma with $20 \%$ tumor cells in bone marrow with an interstitial infiltrative pattern. The lymphoma cells are positive for CD20, PAX5 and cyclin D1; and negative for SOX11 and LEF1 by immunohistochemical stain. Oil immersion, magnification $\times 500$. 
positive. LEF1 was negative in all 19 cases $(0 \%)$ of mantle cell lymphoma assessed.

\section{Cytogenetics and Molecular Findings}

Conventional cytogenetic analysis was performed on bone marrow of 24 patients. Seven $(29 \%)$ had $t$ $(11 ; 14)(q 13 ; q 32)$ and 2 were associated with a complex karyotype. FISH analysis using the IGH/ CCND1 dual color dual fusion was performed in 23 cases; 21 had IGH-CCND1 fusion. In 2 cases that FISH studies were negative due to a low tumor load $(<5 \%)$, cyclin D1 stain studies were positive.

With available materials, molecular analysis of somatic hypermutation of the IGHV genes was performed in four patients (\#3, \#4, \#10 and \#11). All these 4 patients were found positive for IGHV gene mutation. The mutated regions were: \#3: 26 base pair changes in the $V H$ segment $1-8,8.8 \%$ deviation from the germ line sequence; \#4: 7 base pair changes in the VH segment 4-34, 2.6\% deviation; \#10: 13 base pair changes in the VH3, 4.5\% deviation; and \#11: 9 base pair changes in the $V H$ segment $3-7,3.8 \%$ deviation.

\section{Treatment and Outcomes}

The median follow-up of the study group of patients was 23 months (range, 1-183 months). According to mantle cell lymphoma international prognostic index scores calculated from clinical data, 9 patients had a high risk score, 12 intermediate risk, and 4 low risk (Table 2). Twenty-four patients had detailed treatment information available. Of them, 10 patients did not require treatment and had been on observation only, 1 was treated with tonsillectomy only, and two refused chemotherapy and both patients were alive at 6 and 63 months of follow-up. In total, 12 $(50 \%)$ patients did not receive therapy at a median follow-up of 15 months (range, 1-63 months). Of the remaining 12 patients, 9 (36\%) patients received high dose chemotherapy, including two patients with blastoid variant mantle cell lymphoma, 1 patient received rituximab, and 1 patient received ibrutinib and rituximab. For the 11 patients with a leukemic non-nodal presentation, 7 patients did not require treatment and 1 patient received only rituximab monotherapy. Two patients (\#14 and \#15), both with stage IV mantle cell lymphoma, received stem cell transplant (Table 2). At the end of follow-up, 3 patients died including 2 patients with blastoid variant mantle cell lymphoma. The median overall survival was not reached in the present study.

\section{Comparison of Mantle Cell Lymphoma Patients with CD200+ and CD200 - Immunophenotype}

We compared patients with CD200-positive mantle cell lymphoma to a group of 154 well characterized cases of CD200-negative mantle cell lymphoma patients (including 14 blastoid and 7 pleomorphic variant). SOX11 was positive in 69/93 (74\%) cases of CD200-negative mantle cell lymphoma, including 48 strongly positive and 21 partially positive. The frequency of SOX11 expression was significantly lower in CD200-positive mantle cell lymphoma than in CD200-negative mantle cell lymphoma (5/21, 24\% versus 69/93, 74\%, $P<0.0001$ ) (Figure 4a). In contrast, CD23 expression was significantly higher in CD200+ mantle cell lymphoma than CD200negative mantle cell lymphoma $(19 / 25,76 \%$ versus $12 / 154,8 \%, P<0.0001$ ) (Figure $4 \mathrm{~b}$ ). The frequency of CD43 expression in CD200+ mantle cell lymphoma was significantly lower than CD200-negative mantle cell lymphoma $(6 / 22,27 \%$ versus $62 / 115$, $54 \% ; P=0.0346)$.

Patients with CD200+ mantle cell lymphoma more often had a non-nodal leukemic presentation characterized by peripheral blood lymphocytosis and bone marrow involvement and absence of lymphadenopathy or extranodal and/or GI tract disease than patients with CD200-negative mantle cell lymphoma $(11 / 25, \quad 44 \%$ versus $3 / 153,2 \% ; \quad P<0.0001)$ (Figure 4c). In the latter group, 110/154 (71\%) received high dose chemotherapy, significantly higher in CD200-positive mantle cell lymphoma group $(P<0.0001) .22$ patients received other forms of treatment, and therefore a total of 132 (92\%) patients with CD200-negative mantle cell lymphoma needed therapy (Figure 4d). Overall, patients with CD200+ mantle cell lymphoma less often required therapy than patients with CD200-negative mantle cell lymphoma. Overall, among patients with a nonnodal leukemic variant mantle cell lymphoma, CD200 expression was detected in 11/14 (79\%) patients; whereas, of patients with classic nodal/ extranodal based mantle cell lymphoma CD200 was positive in $14 / 164(9 \%)(P<0.001)$.

The overall survival between CD200+ mantle cell lymphoma and CD200-negative patients was not statistically different (Kaplan-Meier log rank test, $P=0.47$ ) (Supplementary Figure 1). The survival was also tested in multivariate analysis considering patients' age, ECOG performance status, WBC, LDH, non-nodal leukemic presentation, CD200, and SOX11 expression. Only ECOG performance status significantly impacted OS $(P=0.003)$ while other factors were not significant. Of note, 2 of the 3 CD200 + patients who died at the end of follow-up were blastoid variant mantle cell lymphoma; and both patients had a very short survival (1 month and 5 months respectively) (Table 2).

\section{Discussion}

With this large cohort of mantle cell lymphoma patients studied by flow cytometry, we show that CD200 is uncommonly expressed in mantle cell lymphoma, in about $3-4 \%$ mantle cell lymphoma 
Table 2 Pathologic findings and clinical outcome of patients of mantle cell lymphoma with CD200 expression

\begin{tabular}{|c|c|c|c|c|c|c|c|c|c|}
\hline Patient \# & Morphology & Involved sites & $\begin{array}{l}\text { Infiltrative } \\
\text { pattern }\end{array}$ & $\begin{array}{l}\text { \% lymphoma } \\
\text { cells in } B M\end{array}$ & SOX11 IHC & Treatment & MIPI scores & $\begin{array}{c}\text { Follow-up } \\
\text { time (months) }\end{array}$ & Status \\
\hline 1 & Classical & $\mathrm{BM}, \mathrm{PB}$ & $\begin{array}{l}\text { Nodular and } \\
\text { interstitial }\end{array}$ & $20-30 \%$ & Negative & $\begin{array}{l}\text { Bortezomib, rituximab, hyper- } \\
\text { CVAD, methotrexate and Ara-C }\end{array}$ & $7.2(\mathrm{HR})$ & 43 & Alive \\
\hline 2 & Small mature & BM, spleen, PB & Interstitial & $20-30 \%$ & Negative & On observation only & 5.2 (LR) & 55 & Alive \\
\hline 3 & Classical & BM, spleen, PB & Interstitial & $70 \%$ & Negative & On observation only & 6.1 (intermediate) & 62 & Alive \\
\hline 4 & Classical & $\mathrm{BM}, \mathrm{PB}$ & Interstitial & $20 \%$ & Negative & On observation only & 5.6 (LR) & 15 & Alive \\
\hline 5 & Small mature & ВM,РВ & Interstitial & $40-50 \%$ & Negative & R-CHOP & 7 (HR) & 28 & Alive \\
\hline 6 & Small mature & $\mathrm{BM}, \mathrm{PB}$ & $\begin{array}{l}\text { Nodular and } \\
\text { interstitial }\end{array}$ & $5 \%$ & Negative & On observation only & 5.8 (intermediate) & 15 & Alive \\
\hline 7 & Classical & BM, spleen, PB & $\begin{array}{l}\text { Nodular and } \\
\text { interstitial }\end{array}$ & $50 \%$ & $\begin{array}{l}\text { Not } \\
\text { performed }\end{array}$ & Ibrutinib and Rituximab & 5.7 (intermediate) & 3 & Alive \\
\hline 8 & Small mature & BM, PB & Interstitial & $20 \%$ & Negative & On observation only & 5.8 (intermediate) & 5 & Unknown \\
\hline 9 & Classical & BM, PB & Interstitial & $20 \%$ & Negative & Rituxan single agent & 5.9 (intermediate) & 42 & Alive \\
\hline 10 & Classical & $\mathrm{BM}, \mathrm{PB}$ & Interstitial & $20 \%$ & Negative & On observation only & 6 (intermediate) & 5 & Alive \\
\hline 11 & Small mature & BM, spleen, PB & Interstitial & $10-20 \%$ & Negative & On observation only & 5.9 (intermediate) & 3 & Alive \\
\hline 12 & Small mature & Skin, BM & Interstitial & $5 \%$ & Negative & Patient refused chemotherapy & 4.6 (LR) & 6 & Alive \\
\hline 13 & Small mature & LN, BM & Interstitial & $5-10 \%$ & Negative & On observation only & 6.2 (HR) & 62 & Alive \\
\hline 14 & Not evaluable & $\begin{array}{l}\text { Submandibular gland, } \\
\text { LN, BM }\end{array}$ & Interstitial & $5 \%$ & $\begin{array}{l}\text { Not } \\
\text { performed }\end{array}$ & $\begin{array}{l}\text { Autologous SCT with BEAM } \\
\text { plus Rituxan }\end{array}$ & $6.2(\mathrm{HR})$ & 45 & Alive \\
\hline 15 & Classical & LN, spleen, BM & $\begin{array}{l}\text { Nodular and } \\
\text { interstitial }\end{array}$ & $40 \%$ & Negative & $\begin{array}{l}\text { R-hyper-CVAD chemotherapy; } \\
\text { allogeneic SCT }\end{array}$ & $6.4(\mathrm{HR})$ & 41 & Alive \\
\hline 16 & Not evaluable & LN, BM, & Interstitial & $5-10 \%$ & $\begin{array}{l}\text { Not } \\
\text { performed }\end{array}$ & On observation only & 6 (intermediate) & 23 & Alive \\
\hline 17 & Small mature & LN, BM, spleen & Interstitial & $5-10 \%$ & Positive & Unknown & 6.7 (HR) & 1 & Alive \\
\hline 18 & Classical & $\mathrm{LN}, \mathrm{BM}$ & $\begin{array}{l}\text { Nodular and } \\
\text { interstitial }\end{array}$ & $50 \%$ & Negative & On observation only & 5.9 (intermediate) & 8 & Alive \\
\hline 19 & Classical & colon, BM, PB & Nodular & $10-20 \%$ & $\begin{array}{l}\text { Not } \\
\text { performed }\end{array}$ & $\begin{array}{l}\text { R-hyper-CVAD, Rituximab/ } \\
\text { Velcade; Revlimid and } \\
\text { thalidomide }\end{array}$ & 6 (intermediate) & 15 & Dead \\
\hline 20 & Classical & GI, BM, PB & Nodular & $5 \%$ & Positive & $\begin{array}{l}\text { Velcade plus R-hyper-CVAD; } \\
\text { Ibrutinib and Rituxan }\end{array}$ & 5.8 (intermediate) & 41 & Alive \\
\hline 21 & Small mature & Tonsil, BM & Nodular & $20 \%$ & Positive & Tonsillectomy only & 6 (intermediate) & 1 & Alive \\
\hline 22 & Classical & Spleen, BM, LN, PB & Nodular & $30-40 \%$ & Negative & On observation only & 7.3 (HR) & 63 & Alive \\
\hline 23 & Classical & Spleen, BM, colon, PB & Interstitial & $20 \%$ & Negative & R-hyper-CVAD & 7.9 (HR) & 62 & Alive \\
\hline 24 & Blastoid & LN, BM, & Nodular & $10-15 \%$ & Positive & Rituxan and hyper-CVAD & 6.7 (HR) & 1 & Dead \\
\hline 25 & Blastoid & LN, GI, BM & Nodular & $30 \%$ & Positive & $\begin{array}{l}\text { R-CHOP, bendamustine and } \\
\text { Rituxan }\end{array}$ & 5 (LR) & 5 & Dead \\
\hline
\end{tabular}

BEAM, carmustine, etoposide, cytarabine and melphalan; BM, bone marrow; GI, gastrointestinal; HR, high risk; LR, low risk; PB, peripheral blood; R-hyper-CVAD, Rituximab, cyclophosphamide, vincristine, doxorubicin, and dexamethasone alternating with methotrexate and Ara-C; R-CHOP, rituximab, cyclophosphamide, doxorubicin, vincristine and prednisone; SCT, stem cell transplant.

*Small subset of lymphoma cells with classical MCL morphology. 

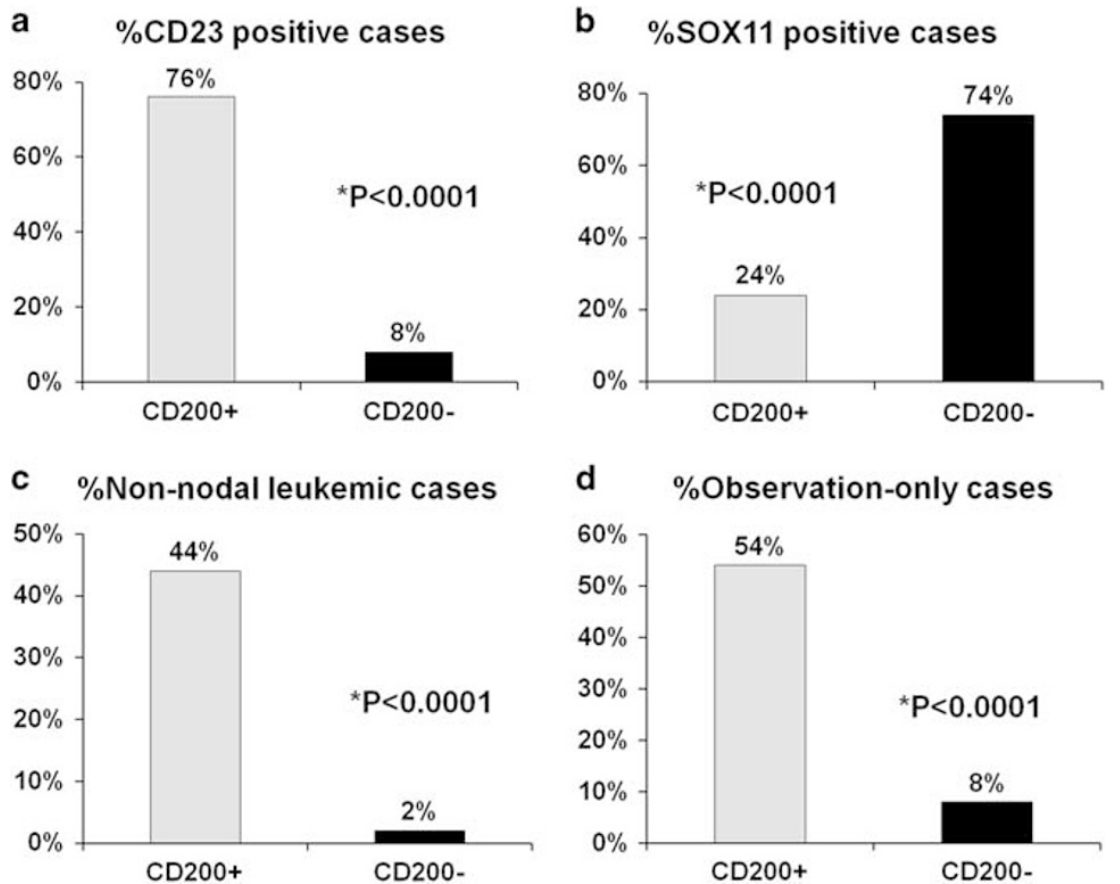

Figure 4 Comparison of pathologic and clinical findings between CD200+ mantle cell lymphoma and randomly chosen CD200- mantle cell lymphoma $(n=154)$. (a) CD23 expression; (b) SOX11 positivity; (c) cases with a non-nodal leukemic presentation; and (d) patients did not require treatment, and were followed with observation only. There are significant differences between these two groups. All $P<0.05$.

patients. We further show that CD200+ mantle cell lymphoma is associated with chronic lymphocytic leukemia -like morphological and immunophenotypic features, frequent $V(H)$ hypermutations and indolent clinical characteristics. These results suggest that assessment of CD200 in mantle cell lymphoma cases might help in identifying indolent subgroups of mantle cell lymphoma cases.

CD200 has been used as an important marker for the differential diagnoses in CD5 positive B-cell lymphoproliferative disorders. ${ }^{10,12,14,16,17}$ In general, CD200 expression is consistently expressed in chronic lymphocytic leukemia cases, while expression of CD200 is highly unusual in mantle cell lymphoma. Palumbo and colleagues studied 14 cases of mantle cell lymphoma by flow cytometry, and observed CD200 expression in $3(21 \%)$ cases, but the expression levels were low and all under $20 \%$ ( $4 \%$ to $16 \%$ ) of lymphoma cells. ${ }^{17}$ In an earlier study by our group that included 61 mantle cell lymphoma cases, three $(5 \%)$ cases showed moderate to bright CD200 expression, with a level similar to normal peripheral blood B cells. ${ }^{16}$ Here we have extended this observation using a large cohort of 668 mantle cell lymphoma patients in whom CD200 was assessed by multicolor flow cytometry immunophenotypic analysis. We observed CD200 expression (in at least $20 \%$ of mantle cell lymphoma cells) in $25(3.7 \%)$ mantle cell lymphoma patients. All 25 patients with CD200+ mantle cell lymphoma had bone marrow involvement. While CD200 was primarily assessed on bone marrow samples, its expression was observed in all 9 peripheral blood samples and 2 lymph node samples that contained mantle cell lymphoma. This finding indicates that CD200 may be unrelated to the bone marrow microenvironment.

We also show that CD23 is frequently positive in CD200+ mantle cell lymphoma cases. This adds to the challenge of distinguishing mantle cell lymphoma from chronic lymphocytic leukemia, since the expression of CD200 and CD23 both support a diagnosis of chronic lymphocytic leukemia. Unlike typical chronic lymphocytic leukemia cases, CD200 + mantle cell lymphoma cases often show a moderate intensity of B-cell markers such as CD20, CD22, and CD79b as well as bright surface immunoglobulin light chain expression; however, there remains substantial overlap with atypical chronic lymphocytic leukemia. Therefore, immunohistochemistry for cyclin D1 expression and cytogenetic analysis for $\mathrm{t}(11 ; 14)(\mathrm{q} 13 ; \mathrm{q} 32) C C N D 1-\mathrm{IGH}$ should be performed in cases not typical for chronic lymphocytic leukemia. Morphologically, all patients with CD200+ mantle cell lymphoma showed bone marrow involvement, with 56\% exhibiting an interstitial pattern, and only $20 \%$ a nodular pattern. Cytologically, $2(9 \%)$ cases showed blastoid morphology, 12 $(52 \%)$ had a classic mantle cell lymphoma morphology, and $9(39 \%)$ cases showed unusual round nuclear contours and clumpy chromatin, with morphological resemblance to chronic lymphocytic leukemia lymphocytes. Due to the overlapping morphological and immunophenotypic features with chronic lymphocytic leukemia, we performed LEF1 (lymphocyte enhancer-binding factor 1) immunohistochemistry stain. LEF1, a crucial transcription 
factor for proliferation and survival of B cells and $\mathrm{T}$ cells, is overexpressed in about $95 \%$ of cases of chronic lymphocytic leukemia. ${ }^{24-26}$ The reported positive LEF1 rate is about $4-9 \%$ in mantle cell lymphoma. ${ }^{25}$ LEF1 was negative in all 19 cases of CD200+ mantle cell lymphoma tested.

Classical mantle cell lymphoma usually develops in IGHV-unmutated or minimally mutated B cells and typically involves lymph nodes and other extranodal sites. A leukemic manifestation associates with a more prognosis of patients with conventional mantle cell lymphoma, upscoring the mantle cell lymphoma prognostic index. ${ }^{22}$ On the other hand, a small subset of mantle cell lymphoma is thought to be derived from IGHV-mutated B cells; and these patients may present with a non-nodal leukemic presentation. ${ }^{2,5,27}$ This latter form of mantle cell lymphoma is recognized by the 2016 WHO revision as a variant of mantle cell lymphoma characterized by lymphocytosis, interstitial bone marrow involvement, and often splenomegaly, but no lymphadenopathy or involvement of extranodal sites. $^{7,28}$ The prevalence of this indolent leukemic variant mantle cell lymphoma is about $3 \%$ of all mantle cell lymphoma. A low frequency (2\%) of non-nodal leukemia presentation was found among the CD200-negative mantle cell lymphoma patients in our cohort. In contrast, 11 (44\%) of the 25 CD200+ mantle cell lymphoma patients met the definition of 'non-nodal leukemic variant mantle cell lymphoma'. Of note, all 4 patients tested showed $V(H)$ hypermutation, further supporting that CD200+ mantle cell lymphoma share a molecular signature with 'nonnodal leukemic variant mantle cell lymphoma' cases. Seven of the 11 patients required no treatment, 2 received single non-chemotherapy treatment, and only 2 received high dose chemotherapy.

SOX11 is expressed in 90-95\% of mantle cell lymphoma cases, including cyclin D1-negative cases, ${ }^{29-31}$ and patients with SOX11 negative mantle cell lymphoma often have been noted to carry an indolent clinical course. ${ }^{3}$ Furthermore, the nonnodal leukemic variant mantle cell lymphoma is often SOX11 negative. ${ }^{6,7,28}$ In our patients, SOX11 was negative in all 9 non-nodal leukemic variant mantle cell lymphoma cases. Interestingly, of 13 patients with lymph node or extranodal disease who would be considered to have stage IV disease, SOX11 was negative in 6 patients. All 6 of these patients did not require high dose chemotherapy. It is noteworthy that over the course of the disease of mantle cell lymphoma, including non-nodal leukemic variant indolent mantle cell lymphoma, additional molecular/cytogenetic abnormalities can be acquired and lead to more aggressive forms of mantle cell lymphoma, ${ }^{32,33}$ which might account for some aggressive disease in this cohort of patients. One of the two blastoid variant mantle cell lymphoma tested was positive for p53 by immunohistochemistry.

The biology underlying the finding that CD200 expression in mantle cell lymphoma correlates with unique pathological features and an indolent clinical behavior is not known. The entity of 'monoclonal asymptomatic lymphocytosis, cyclin D1-positive' likely corresponds to the currently recognized indolent non-nodal leukemic variant mantle cell lymphoma. Espinet and colleagues speculated that an increased frequency of inhibitory signals emerging from CD200 to CD200R (an inhibitory receptor expressed by both lymphoid and myeloid cells) attenuates the proliferation of monoclonal asymptomatic lymphocytosis, cyclin D1-positive B cells, and contributes to its indolent behavior. ${ }^{18}$ We believe that these indolent variants of mantle cell lymphoma should be recognized in the pathology report and patients with this disease should be managed differently from the classical mantle cell lymphoma and avoid overtreatment.

In summary, CD200 expression in mantle cell lymphoma is uncommon $(\sim 4 \%)$, and correlates with a higher frequency of CD23 expression and small round cell morphology, features that overlap with chronic lymphocytic leukemia. These findings indicate the importance of assessing for cyclin D1 expression and CCND1-IGH rearrangement in CD5+ lymphoma/leukemia. CD200 expression identifies a subset of mantle cell lymphoma patients in whom SOX11 is frequently negative, likely associated with $V(H)$ hypermutations and patients present with nonnodal leukemic disease, an indolent clinical course that may not need immediate therapy. These findings indicate that CD200 expression is useful to identify patients with mantle cell lymphoma undergoing an indolent course.

\section{Acknowledgments}

We thank Marisol Gonzalez for her administrative assistance.

\section{Disclosure/conflict of interest}

The authors declare no conflict of interest.

\section{References}

1 Swerdlow SH, Campo E, Seto M, et al. Mantle cell lymphoma. In: Swerdlow SH, Campo E, Harris NL, et al. eds. WHO Classification of Tumours of Haematopoietic and Lymphoid Tissues. 4th edn. France, Lyon, 2008, pp 229-232.

2 Dreyling M, Amador V, Callanan M, et al. Update on the molecular pathogenesis and targeted approaches of mantle cell lymphoma: summary of the 12th annual conference of the EuropeanMantle Cell Lymphoma Network. Leuk Lymphoma 2015;56:866-876.

3 Fernandez V, Salamero O, Espinet B, et al. Genomic and gene expression profiling defines indolent forms of mantle cell lymphoma. Cancer Res 2010;70:1408-1418.

4 Furtado M, Rule S. Indolent mantle cell lymphoma. Haematologica 2011;96:1086-1088. 
5 Jares P, Colomer D, Campo E. Molecular pathogenesis of mantle cell lymphoma. J Clin Invest 2012;122: 3416-3423.

6 Sander B, Quintanilla-Martinez L, Ott G, et al. Mantle cell lymphoma-a spectrum from indolent to aggressive disease. Virchows Arch 2016;468:245-257.

7 Swerdlow SH, Campo E, Pileri SA, et al. The 2016 revision of the World Health Organization classification of lymphoid neoplasms. Blood 2016;127:2375-2390.

8 Barclay AN, Clark MJ, McCaughan GW. Neuronal/ lymphoid membrane glycoprotein MRC OX-2 is a member of the immunoglobulin superfamily with a light-chain-like structure. Biochem Soc Symp 1986;51: 149-157.

9 Wright GJ, Jones M, Puklavec MJ, et al. The unusual distribution of the neuronal/lymphoid cell surface CD200 (OX2) glycoprotein is conserved in humans. Immunology 2001;102:173-179.

10 Alapat D, Coviello-Malle J, Owens R, et al. Diagnostic usefulness and prognostic impact of CD200 expression in lymphoid malignancies and plasma cell myeloma. Am J Clin Pathol 2012;137:93-100.

11 Brunetti L, Di Noto R, Abate G, et al. CD200/OX2, a cell surface molecule with immuno-regulatory function, is consistently expressed on hairy cell leukaemia neoplastic cells. Br J Haematol 2009;145:665-667.

12 El Desoukey NA, Afify RA, Amin DG, et al. CD200 expression in B-cell chronic lymphoproliferative disorders. J Investig Med 2012;60:56-61.

13 Pillai V, Pozdnyakova O, Charest K, et al. CD200 flow cytometric assessment and semiquantitative immunohistochemical staining distinguishes hairy cell leukemia from hairy cell leukemia-variant and other B-cell lymphoproliferative disorders. Am J Clin Pathol 2013;140:536-543.

14 Sandes AF, de Lourdes Chauffaille M, Oliveira CR, et al. CD200 has an important role in the differential diagnosis of mature B-cell neoplasms by multiparameter flow cytometry. Cytometry B Clin Cytom 2014;86:98-105.

15 Wong KK, Khatri I, Shaha S, et al. The role of CD200 in immunity to B cell lymphoma. J Leukoc Biol 2010;88: 361-372.

16 Challagundla P, Medeiros LJ, Kanagal-Shamanna R, et al. Differential expression of CD200 in B-cell neoplasms by flow cytometry can assist in diagnosis, subclassification, and bone marrow staging. Am J Clin Pathol 2014;142:837-844.

17 Palumbo GA, Parrinello N, Fargione G, et al. CD200 expression may help in differential diagnosis between mantle cell lymphoma and B-cell chronic lymphocytic leukemia. Leuk Res 2009;33:1212-1216.

18 Espinet B, Ferrer A, Bellosillo B, et al. Distinction between asymptomatic monoclonal B-cell lymphocytosis with cyclin D1 overexpression and mantle cell lymphoma: from molecular profiling to flow cytometry. Clin Cancer Res 2014;20:1007-1019.
19 Yabe M, Medeiros LJ, Wang SA, et al. Distinguishing between hepatosplenic T-cell lymphoma and gammadelta T-cell large granular lymphocytic leukemia: a clinicopathologic, immunophenotypic, and molecular analysis. Am J Surg Pathol 2017;41:82-93.

20 Onciu M, Schlette E, Medeiros LJ, et al. Cytogenetic findings in mantle cell lymphoma cases with a high level of peripheral blood involvement have a distinct pattern of abnormalities. Am J Clin Pathol 2001;116: 886-892.

21 Shaffer L, Mj J, Schmid M. International System for Human Cytogenetic Nomenclature. Karger: Basal, Switzerland, 2013.

22 Hoster E, Dreyling M, Klapper W, et al. A new prognostic index (MIPI) for patients with advancedstage mantle cell lymphoma. Blood 2008;111:558-565.

$23 \mathrm{Hu} \mathrm{Z}$, Medeiros LJ, Chen Z, et al. Mantle cell lymphoma with MYC rearrangement: a report of 17 patients. Am J Surg Pathol 2017;41:216-224.

24 Menter T, Dirnhofer S, Tzankov A. LEF1: a highly specific marker for the diagnosis of chronic lymphocytic B cell leukaemia/small lymphocytic B cell lymphoma. J Clin Pathol 2015;68:473-478.

25 O'Malley DP, Lee JP, Bellizzi AM. Expression of LEF1 in mantle cell lymphoma. Ann Diagn Pathol 2017;26: 57-59.

26 Tandon B, Peterson L, Gao J, et al. Nuclear overexpression of lymphoid-enhancer-binding factor 1 identifies chronic lymphocytic leukemia/small lymphocytic lymphoma in small B-cell lymphomas. Mod Pathol 2011;24:1433-1443.

27 Vogt N, Dai B, Erdmann T, et al. The molecular pathogenesis of mantle cell lymphoma. Leuk Lymphoma 2016;1-8.

28 Ondrejka SL, Lai R, Smith SD, et al. Indolent mantle cell leukemia: a clinicopathological variant characterized by isolated lymphocytosis, interstitial bone marrow involvement, kappa light chain restriction, and good prognosis. Haematologica 2011;96:1121-1127.

29 Ferrando AA. SOX11 is a mantle cell lymphoma oncogene. Blood 2013;121:2169-2170.

30 Palomero J, Vegliante MC, Eguileor A, et al. SOX11 defines two different subtypes of mantle cell lymphoma through transcriptional regulation of BCL6. Leukemia 2016;30:1596-1599.

31 Vegliante MC, Palomero J, Perez-Galan P, et al. SOX11 regulates PAX5 expression and blocks terminal B-cell differentiation in aggressive mantle cell lymphoma. Blood 2013;121:2175-2185.

32 Gallo M, Cacheux V, Vincent L, et al. Leukemic nonnodal mantle cell lymphomas have a distinct phenotype and are associated with deletion of PARP1 and 13q14. Virchows Arch 2016;469:697-706.

33 Chapman-Fredricks J, Sandoval-Sus J, Vega F, et al. Progressive leukemic non-nodal mantle cell lymphoma associated with deletions of TP53, ATM, and/or 13q14. Ann Diagn Pathol 2014;18:214-219.

Supplementary Information accompanies the paper on Modern Pathology website (http://www.nature.com/ modpathol) 